\title{
Increased salivary level of 8-hydroxydeoxyguanosine is a marker of premature oxidative mitochondrial DNA damage in gingival tissue of patients with periodontitis
}

\author{
Cenk Fatih Çanakçi ${ }^{1}$, Varol Çanakçi ${ }^{1}$, Abdulgani Tatar $^{2}$, Abubekir Eltas $^{1}$, Ufuk Sezer ${ }^{3}$, \\ Yasin Çiçek ${ }^{1}$ and Sitki Oztas ${ }^{2}$ \\ ${ }^{1}$ Department of Periodontology, Ataturk University, Faculty of Dentistry, Erzurum, Turkey \\ ${ }^{2}$ Department of Genetics, Faculty of Medicine, Ataturk University, Erzurum, Turkey \\ ${ }^{3}$ Department of Periodontology, Karadeniz Technical University, Faculty of Dentistry, Trabzon, Turkey
}

Received: 2008.10.19, Accepted: 2009.01.28, Published online: 2009.05.29

(C) L. Hirszfeld Institute of Immunology and Experimental Therapy, Wrocław, Poland 2009

\begin{abstract}
Introduction: Oxidative stress may contribute to the pathogenesis of periodontitis. However, the detailed molecular mechanism remains unclear. Both 8-hydroxydeoxyguanosine (8-OHdG) and mitochondrial DNA (mtDNA) deletion have been reported as early oxidative DNA damage markers. In this study, 8-OHdG levels in saliva and mtDNA deletions in gingival tissue of patients with chronic periodontitis $(\mathrm{CP})$ were evaluated.

Materials and Methods: Gingival tissue and whole saliva samples were collected from 32 patients with CP and 32 healthy control subjects. To determine the clinical condition of each subject, the plaque index, gingival index, clinical attachment level (CAL), and probing depth (PD) were measured. Using the ELISA and polymerase chain reaction methods, the salivary 8-OHdG levels and the 7.4-kbp and 5-kbp mtDNA deletions were examined.

Results: The 5-kbp mtDNA deletion was detected in 20 of the 32 periodontitis patients $(62.5 \%)$, but was not detected in the healthy controls. The mean value of $8-\mathrm{OHdG}$ in the saliva of the periodontitis patients with deleted mtDNA was significantly higher than in the patients with non-deleted mtDNA $(\mathrm{p}<0.01)$. Also, significant correlation was found between the occurrence of the 5-kbp mtDNA deletion and salivary 8-OHdG levels $(\mathrm{p}<0.01)$. Similar correlations were detected between salivary 8 -OHdG levels and age, $\mathrm{PD}$, and $\mathrm{CAL}(\mathrm{p}<0.01, \mathrm{p}<0.05)$.

Conclusion: Increased oxidative stress may lead to premature oxidative DNA damage in the gingival tissue of periodontitis patients and the salivary 8-OHdG level may signify premature oxidative mtDNA damage in diseased gingival tissue.
\end{abstract}

Key words: periodontitis, gingiva, saliva, 8-hydroxyguanosine, mitochondrial DNA deletion, oxidative DNA damage.

Corresponding author: Dr. Cenk Fatih Çanakçi, Atatürk Üniversitesi, Diş Hekimligi Fakültesi, Periodontoloji Anabilim Dali, 25240 Erzurum, Turkey, fax: +90 442-2360945, e-mail: cfcanakci@yahoo.com

\section{INTRODUCTION}

Oxidative stress is defined as the condition occurring when the physiological balance between oxidants and antioxidants is disrupted in favor of the former with potential damage for the organism (Sies 1991). The deleterious effects of increased oxidative stress are termed oxidative damage and generally occur after exposure to a relatively high concentration of reactive oxygen species (ROS) and/or a decrease in the antioxidant (AO) defense system against ROS. Free radicals and related species have well-defined roles in the inflammatory process (Canakci et al. 2005). ROS and ROS-derived radical formations can cause extensive damage to proteins, lipids, and DNA molecules and have been implicated as a major contributor in over 100 disorders and, more recently, periodontitis (Canakci et al. 2005).

Periodontitis is an oral inflammatory disorder affecting alveolar bone, gingiva, and periodontal ligament initiated by the complex interaction between the presence of microorganisms (periodontopathic bacteria) and the host's immune response. The exact mechanism of periodontitis' etiopathogeny and pathophysiology is not 
clear. Several lines of evidence implicate polymorphonuclear cells (PMNs) as the primary mediators of the host response against periodontopathic bacteria (Miller et al. 1984). A study carried out by Moseley et al. (Moseley et al. 1997) demonstrates that PMNs produce a range of antimicrobial factors, including ROS, during the phagocytosis of periodontopathic bacteria. ROS have recently been reviewed in the pathogenesis of periodontitis (Canakci et al. 2005). It has been suggested that as a result of stimulation by bacterial antigens, PMNs produce and release a large quantity of ROS, culminating in heightened oxidative damage to gingival tissue, periodontal ligament, and alveolar bone (Sculley and Langley-Evans 2002). ROS are active in the depolymerization of extracellular matrix components, lipid peroxidation, oxidation of enzymes such as anti-proteases, induction of pro-inflammatory cytokines, and DNA damage (Canakci et al. 2005; Ozmeric 2005).

Oxidative stress can result in DNA damage, including the oxidation of nucleosides. 8-hydroxydeoxyguanosine (8-OHdG) is an oxidized nucleoside that is excreted in the bodily fluids with DNA repair. Several studies have demonstrated that $8-\mathrm{OHdG}$ in bodily fluids can act as a biomarker of oxidative stress (Chiou et al. 2003; Liu et al. 2004; Wu et al. 2004) and 8-OHdG is commonly used as a marker to evaluate oxidative DNA damage in disorders including chronic inflammatory diseases (Chapple and Matthews 2007).

The human mitochondrial genome is a circular double-stranded DNA molecule composed of 16,569 base pairs. In each cell there are a few hundred to thousands of mitochondria, and each mitochondrion contains 2-10 copies of mitochondrial DNA (mtDNA) that encodes 13 genes essential for OXPHOS, 2 ribosomal RNA genes, and transfer RNA (tRNA) genes required for mtDNA expression. Human mtDNA is naked and is located in the vicinity of mitochondrial inner membranes, where abundant ROS and free radicals are continually generated. It is not protected by histones and replicates faster than nuclear DNA, without proofreading or an efficient DNA repair systems (Yakes and van Houten 1997). In the last decade a number of pathogenic mutations of mtDNA, such as large-scale deletions and point mutations, have been established as responsible for or associated with several distinct human diseases (Wallace et al. 1995). More than 100 large-scale deletions have been identified in various tissues of human subjects with specific mitochondrial diseases, degenerative diseases, and aging (Lee et al. 1994). These large-scale deletions often cause the removal or truncation of multiple structural genes and tRNA genes of the mitochondrial genome, which can result in multiple respiratory-chain deficiencies. Among these deletions, a 4977-bp deletion, termed the "5-kbp common deletion", is most frequently seen in various tissues of aged individuals (Yen et al. 1991).

In the present study we hypothesized that increased level of salivary $8-\mathrm{OHdG}$ may reflect oxidative damage to the mtDNA of gingival tissue in periodontitis patients. We investigated the salivary $8-\mathrm{OHdG}$ levels and mtDNA deletions of gingival tissue in periodontitis patients and healthy controls and explored correlations between these oxidative DNA damage markers and clinical parameters of periodontitis and age.

\section{MATERIALS AND METHODS}

\section{Study groups}

The study was carried out in 64 subjects: 32 patients with chronic periodontitis (CP) (16 males and 16 females, age range: $35-72$, mean age: 48.7 years) and 32 periodontally healthy controls (16 males and 16 females, age range: $25-69$, mean age: 45.4 years). The subjects were selected from among individuals who applied to the Ataturk University Faculty of Dentistry, Department of Periodontology, due to periodontal problems or for routine check-ups. The criteria for CP were judged from standard measurements of clinical probing depths (PDs) and radiographic bone loss according to currently accepted criteria (Armitage 1999). Subjects with CP had at least two sides showing a PD of $>4 \mathrm{~mm}$, gingival inflammation, and $30 \%$ bone loss. The control group was composed of individuals with no history of any periodontal disease, with no gingival inflammation, and with good oral hygiene. The subjects included in the study had no history of systemic disease, had not received periodontal therapy, had not taken antibiotics, antiinflammatory drugs, or any other drugs for at least six months, were never-smokers, and were not alcohol or AO consumers. This study was approved by the ethics committee of Ataturk University and written informed consent was obtained from each participant.

Periodontal status of the tissue sampling areas in all subjects was determined by measuring the plaque index (PI) (Silness and Löe 1964), gingival index (GI) (Löe and Silness 1963), PD, and clinical attachment level (CAL). PD and CAL were measured on six sites of teeth. Full-mouth periapical radiographs were taken to determine the level of periodontal bone loss of the patients.

\section{Collection of samples}

Diseased gingival tissues with periodontal pockets $\geq 5 \mathrm{~mm}$, gingival inflammation, and bone loss were collected during periodontal flap surgery from the $\mathrm{CP}$ group and healthy gingival tissues were collected during crown lengthening procedures. All tissue samples were obtained from the gingival margin area. Each sample was transferred to a $50-\mathrm{ml}$ falcon tube with $10 \mathrm{ml}$ of phosphate-buffered solution. Whole saliva samples were collected under resting conditions in a quiet room between 9 a.m. and noon at least $8 \mathrm{~h}$ after food intake and were obtained by expectorating into disposable tubes before clinical measurements. About $2 \mathrm{ml}$ of whole saliva was collected in tubes and centrifuged 
immediately to remove cell debris $(1000 \times \mathrm{g}$ for $10 \mathrm{~min}$ at $\left.4^{\circ} \mathrm{C}\right)$. The supernatant was removed and stored in small aliquots at $-80^{\circ} \mathrm{C}$ until analysis.

\section{DNA extraction}

Each tissue sample underwent centrifugation for 5 $\min$ at $3000 \times \mathrm{g}$ and the supernatant was removed. The pellet was resuspended in $200 \mu \mathrm{l}$ of TE buffer and total DNA of the tissues was extracted by proteinase K/SDS lysis followed by phenol/chloroform extraction as previously described (Liu et al. 1996). After ethanol precipitation, the DNA was dissolved in $100 \mu \mathrm{l}$ of distilled water and frozen at $-30^{\circ} \mathrm{C}$ until use.

\section{Polymerase chain reaction}

Each desired DNA fragment was amplified in $50 \mu \mathrm{l}$ of reaction mixture containing $16 \mu \mathrm{l}$ of DNA $(1 \mu \mathrm{g} / 50$ $\mu \mathrm{l}), 1.5 \mu \mathrm{l}$ of deoxynucleotide triphosphate $(0.2 \mathrm{mM})$ (MBI Fermentas, St. Leon-Rot, Germany), $1.5 \mu \mathrm{l}$ of each primer $(1 \mu \mathrm{M}), 1.5 \mu \mathrm{l}$ of Taq DNA polymerase $(5$ $\mathrm{U} / 50 \mu \mathrm{l})$ (Taq, MBI Fermentas), $3 \mu \mathrm{l}$ of $\mathrm{MgCl}_{2}$ (4 mM/50 $\mu \mathrm{l}$ ), $5 \mu \mathrm{l}$ of 10XPCR buffer (without magnesium; MBI Fermentas), and $20 \mu \mathrm{l}$ of sterilized water. The reactions were carried out for 30 cycles in a Sanyo DNA Amplifier thermal cycler (MIR-D30, Sanyo, Tokyo, Japan). The polymerase chain reaction (PCR) cycles consisted of 25 s denaturation at $94^{\circ} \mathrm{C}, 25 \mathrm{~s}$ annealing at $50^{\circ} \mathrm{C}$, and $80 \mathrm{~s}$ primer extension at $72^{\circ} \mathrm{C}$. The PCR products were separated on a $1.5 \%$ agarose gel at $110 \mathrm{~V}$ for $40 \mathrm{~min}$ and were detected fluorographically after staining with $0.5 \mu \mathrm{g} / \mathrm{ml}$ ethidium bromide (Sigma-Aldrich, Deisenhofen, Germany). The gel patterns of the PCR products were photographed under UV transillumination (Syngene, Cambridge, UK).

\section{Determination of the proportion of deleted $m t D N A$}

For determination of the deleted mtDNA we employed a semiquantitative PCR method as previously described (Lee et al. 1994). Each of the mtDNA samples was serially diluted by twofold with distilled water before PCR amplification. The range of dilution was usually between $2^{0}$ and $2^{22}$. Three different pairs of PCR primers (Thermo Electron Corp., MA, USA) were used in this study. The primers L[6251] 5'-TATAGTGGAGGCCGGAGCAG-3' and H[7261] 5'-GAATGAGCCTACAGATGATA-3' were used for amplification of a 1.0-kbp DNA fragment from the total mtDNA. The primers L[6251] and H[7261] were used for proving DNA extraction and the PCR procedures. We used the primers L[7901]5'-TGAACCTACGAGTACACCGA-3'and H[13631] 5' GGGGAAGCGAG GTTGACCTG-3' for amplification of a $0.8-\mathrm{kbp}$ PCR product from the 5-kbp deleted mtDNA. Meanwhile, we used the primers L[8531]5'-ACGAAAATCTGTTC GCTTCA-3' and H[381] 5'AAATTTGAAATCTGGTTA GG-3' for amplification of a $1.0-\mathrm{kbp}$ PCR product from the 7.4- kbp deleted mtDNA. In addition, two different DNA ladders (Phix 174, Sigma-Aldrich; GeneRuler 100-bp -DNA ladder, MBI Fermentas) were used for determination of the DNA fragment size.

\section{Determination of salivary 8-OHdG using ELISA}

Saliva samples were centrifuged at $10,000 \times \mathrm{g}$ for 10 min and the supernatant was used to determine the $8-\mathrm{OHdG}$ levels with a competitive ELISA kit (8-OHdG Check, Highly Sensitive 8-OHdG Check, Japan Institute for the Control of Aging, Shizuoka, Japan) Levels ranged from 0.125 to $200 \mathrm{ng} / \mathrm{ml}$.

\section{Statistical analyses}

Differences in clinical parameters between the $\mathrm{H}$ and CP groups were analyzed by an unpaired Student's $t$-test. Differences in salivary $8-\mathrm{OHdG}$ levels between the $\mathrm{H}$ and $\mathrm{CP}$ groups were analyzed by the Mann-Whitney U-test. Correlation between salivary 8-OHdG levels, clinical parameters, and the presence of mtDNA deletion were analyzed by the Spearman rank correlation test. All statistical analyses were performed using statistical software (SPSS 13.0 for Windows, SPSS, Chicago, IL, USA).

\section{RESULTS}

\section{Clinical findings}

The median values of the clinical parameters are shown in Table 1. All clinical parameter scores of the tissue sampling areas were significantly higher in the $\mathrm{CP}$ group than in the control group $(\mathrm{p}<0.001)$. There was no significant difference between the mean ages of the two groups $(\mathrm{p}>0.05)$.

\section{Laboratory findings}

Table 1. Clinical parameters of the groups (mean \pm SD)

\begin{tabular}{lcc}
\hline & Controls & CP group \\
\hline $\mathrm{N}$ & 32 & 32 \\
\hline Age & $45.40 \pm 11.9$ & $48.70 \pm 9.30$ \\
$\mathrm{PI}$ & $0.78 \pm 0.41$ & $1.81 \pm 0.52^{*}$ \\
$\mathrm{GI}$ & $0.61 \pm 0.37$ & $1.76 \pm 0.43^{*}$ \\
PD $(\mathrm{mm})$ & $2.03 \pm 0.45$ & $4.33 \pm 0.78^{*}$ \\
CAL $(\mathrm{mm})$ & $0.00 \pm 0.00$ & $4.62 \pm 1.01^{*}$ \\
\hline
\end{tabular}

"Difference is significant $(\mathrm{p}<0.001)$.

Abbreviations: $\mathrm{N}$ - number of subjects, PI - plaque index, GI - gingival index, PD - probing depth, CAL - clinical attachment level. 
Table 2. mtDNA deletions of the groups (n \%)

\begin{tabular}{llll}
\hline & Control region & 5-kbp deletion region & 7.4-kbp deletion region \\
\hline Primers & L[6251], H[7261] & L[7901], H[13631] & L[8531], H[381] \\
\hline Fragment & $\approx 1.0 \mathrm{kbp}$ & $\approx 0.8 \mathrm{kbp}$ & $\approx 1.0 \mathrm{kbp}$ \\
\hline Controls & $32(+) 100 \%$ & $0(+) 0 \%$ & $0(+) 0 \%$ \\
& $0(-) 0 \%$ & $32(-) 100 \%$ & $32(-) 100 \%$ \\
\hline CP group & $32(+) 100 \%$ & $20(+) 62.5 \%$ & $0(+) 0 \%$ \\
& $0(-) 0 \%$ & $12(-) 37.5 \%$ & $32(-) 100 \%$ \\
\hline
\end{tabular}

PCR was performed using three primer pairs for three regions (control region, 5-kbp deletion region, and 7.4-kbp deletion region) as described in Materials and Methods. The primer pair of L[6251] and H[7261] was used for amplification of a 1.0-kbp DNA fragment from the total mtDNA and for proving of DNA extraction and of PCR procedures. In the chronic periodontitis (CP) and control groups, approx. 1.0-kbp DNA fragment was observed in all individuals (32(+) 100\% in both groups). The primer pair of L[7901] and $\mathrm{H}[13631]$ was used for amplification of a $0.8-\mathrm{kbp}$ DNA fragment from the total mtDNA and used for determination of the 5kbp deletion. In the CP group, approx. 0.8-kbp DNA fragment was observed in 20 of the 32 subjects $(20(+) 62.5 \%, 12(-) 37.5 \%)$. In the controls, approx. 0.8-kbp DNA fragment was not observed (32(-) 100\%). The primer pair of L[8531] and H[381] were used for amplification of a 1.0-kbp DNA fragment from the total mtDNA and used for determination of 7-kbp deletion. In the CP and control groups, approx. 1.0-kbp DNA fragment was not observed (32(-) 100\% in both groups).

The 5-kbp mtDNA deletion was observed in 20 of the 32 periodontitis patients and was not detected in the controls (Table 2, Fig. 1). This result demonstrates that the frequency of occurrence of the 5-kbp mtDNA deletion in the CP group was $62.5 \%$. On the other hand, the 7.4-kbp mtDNA deletion was not detected in the gingival tissues of the subjects.

The mean 8-OHdG level in the saliva of the periodontally diseased patients in the CP group $(3.76 \pm 0.30$ $\mathrm{ng} / \mathrm{ml})$ was significantly higher $(\mathrm{p}<0.01)$ than that of the controls $(1.41 \pm 0.22 \mathrm{ng} / \mathrm{ml})$. In the CP group, the salivary 8 -OHdG levels of the patients with deleted mtDNA were significantly higher than those with nondeleted mtDNA ( $p<0.01$, Fig. 2). However, the salivary 8 -OHdG levels of the periodontitis patients with non-deleted mtDNA were not significantly higher than those of the healthy controls $(p>0.05)$.

\section{Correlation of the 5-kbp mtDNA deletion with clinical parameters and salivary 8-OHdG levels in the CP group}

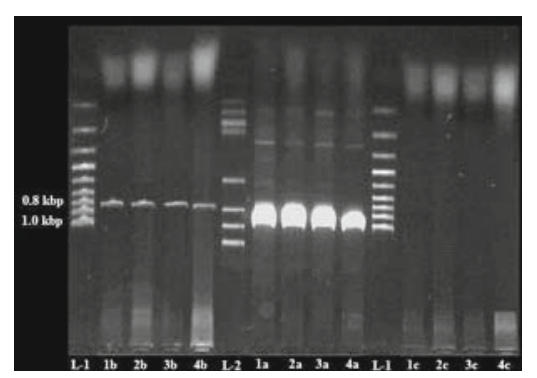

Fig. 1. A photograph of $1.5 \%$ agarose gel of four patients with chronic periodontitis (patient numbers 1, 2, 3, 4). L-1: 100-bp DNA ladder. L-2: Phix 174-bp DNA ladder. The mtDNA extraction and PCR protocol were confirmed by using controls $(\approx 1.0-\mathrm{kbp}$ fragments at lanes of $1 \mathrm{a}, 2 \mathrm{a}, 3 \mathrm{a}$, and $4 \mathrm{a}$ ). The $5 \mathrm{kp}$ mtDNA deletions are detected $(\approx 0.8$-kbp fragments at lanes of $1 \mathrm{~b}, 2 \mathrm{~b}, 3 \mathrm{~b}$, and $4 \mathrm{~b}$ ). The 7.4-kp mtDNA deletion is not detected (no fragment in lanes $1 \mathrm{c}, 2 \mathrm{c}, 3 \mathrm{c}$, and $4 \mathrm{c}$ ).
We evaluated the relationships among the occurrence of the 5-kbp mtDNA deletion, clinical parameters (PI, GI, PD, CAL, and age), and salivary 8-OHdG levels in the CP group. Statistically significant positive correlations were observed between the occurrence of the 5-kbp mtDNA deletion and age, PD, and CAL. Similarly, the salivary levels of 8-OHdG correlated with age, PD, and CAL $(\mathrm{p}<0.01, \mathrm{p}<0.05$; Table 3). Additionally, a positive correlation was found between the occurrence of the 5-kbp mtDNA deletion and salivary $8-O H d G$ levels $(p<0.01)$.

\section{DISCUSSION}

Both the level of 8-OHdG and the mtDNA mutation are superb markers of oxidative DNA damage. Oxidative DNA damage is reported to be involved in the pathogenesis of many chronic conditions, including neurodegenerative disease (Wang et al. 2008), diabetes (Kakimoto et al. 2002), cancer (Wada et al. 2006), and chronic inflammatory conditions (Dincer et al. 2007). However, information on markers of ROS reactions with DNA in periodontitis is limited. The majority of published data on oxidative damage to DNA has been reported by a Japanese group who investigated $8-\mathrm{OHdG}$ levels in the saliva of periodontitis patients (Sawamoto et al. 2005; Takane et al. 2005; Takane et al. 2002). Their studies demonstrated that salivary levels of $8-\mathrm{OHdG}$ in samples from subjects with $\mathrm{CP}$ were significantly higher than those from periodontally healthy controls. Interestingly, increasing salivary 8-OHdG level may be a biomarker of the oxidative damage of nuclear DNA, mtDNA, or both; thus previous periodontal studies on salivary 8 -OHdG levels did not mention that oxidative DNA damage may strongly depend on a damage tendency of mtDNA in inflamed gingival tissue of periodontitis patients. In our previous study (Canakci et al. 


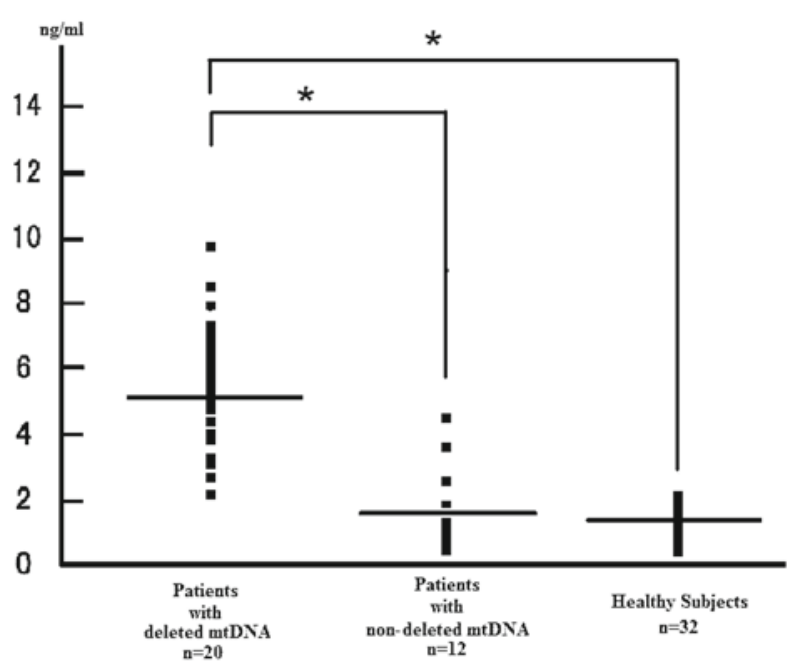

Fig. 2. Distribution of the salivary $8-\mathrm{OHdG}$ levels from the periodontitis patients and healthy subjects. The periodontitis patients were divided into two subgroups based on the presence/absence of mtDNA deletion in gingival tissues. Salivary 8-OHdG levels: $1.41 \pm 0.12$ in healthy subjects, $1.61 \pm 0.22$ in patients with non-deleted mtDNA, and $5.01 \pm 0.17$ in patients with deleted mtDNA. (mean \pm SE $\mathrm{ng} / \mathrm{ml})$. Difference is significant $\left({ }^{*} \mathrm{p}<0.01\right.$, Mann-Whiteny's U-test).

2006) we detected the 5-kbp mtDNA deletion in gingival tissues of periodontitis patients and its frequency of occurrence was approximately $80 \%$. We also suggested that because mtDNA contains higher steady-state amounts of oxidative DNA damage than nuclear DNA, the extracellular overproduction of ROS by activated PMNs in chronic inflammation may lead primarily to premature oxidative damage of the mtDNA in nearby cells.

In this study we measured salivary $8-\mathrm{OHdG}$ levels and examined mtDNA deletions of gingival tissues in periodontitis patients and healthy controls. Our study showed that the salivary $8-\mathrm{OHdG}$ levels were significantly higher in the periodontitis patients than in the healthy controls. This result confirms findings of limited previous studies (Sawamoto et al. 2005; Takane et al. 2005; Takane et al. 2002). Also, we detected the 5-kbp mtDNA deletion in the gingival tissues of 20 of the 32 periodontitis patients and did not detect the 7-kbp
mtDNA deletion in the periodontitis patients. This result parallels the result of our previous study (Canakci et al. 2006). The 5-kbp mtDNA deletion is known as a "common deletion" and the one most frequently observed. It is about 4977 bp in length and occurs at a presumed deletion "hot spot" involving two 13-base direct repeats. Unfortunately, the reasons for the high incidence of the 5-kbp mtDNA deletion are not clear. It is suggested that both the incidence and abundance of the 5-kbp mtDNA deletion tend to be higher in tissues with a high metabolic rate (Liu et al. 1998). The very specific metabolic function of inflamed gingival tissue cells may be the reason why the abundance of the 5-kbp mtDNA deletion is higher than that of the 7-kbp mtDNA deletion in periodontitis patients. Notably, we first determined that the salivary 8 -OHdG levels of periodontitis patients with deleted mtDNA were significantly higher than those of patients with non-deleted mtDNA and that there was a positive correlation between salivary 8-OHdG level and the occurrence of the 5-kbp mtDNA deletion in diseased gingival tissues. Similar correlations were previously reported by Kakimoto et al. (Kakimoto et al. 2002) and Suzuki et al. (Suzuki et al. 1999) in their studies on the association between oxidative DNA damage and diabetes.

Takane et al. (Takane et al. 2002) reported that salivary 8 -OHdG level may be a useful marker for periodontitis. In another study carried out by Takane et al. (Takane et al. 2004) the authors found significantly higher salivary 8-OHdG levels in subjects with periodontally hopeless teeth (teeth with loss of over $75 \%$ bone support and PDs greater than $8 \mathrm{~mm}$ ) than in subjects without periodontally hopeless teeth and in clinically healthy controls, but the authors somehow did not show data on the relationship between salivary $8-\mathrm{OHdG}$ levels in subjects without periodontally hopeless teeth and in clinically healthy controls. In the present study the salivary 8-OHdG levels of periodontitis patients with non-deleted mtDNA were not significantly higher than those of healthy controls. Therefore the findings in this study indicate that increased salivary $8-\mathrm{OHdG}$ levels reflect premature oxidative mtDNA deletion of gingiva in periodontitis patients. However, we are not saying that salivary $8-\mathrm{OHdG}$ level may be a marker for all cases of periodontitis.

Table 3. Correlation between salivary 8-OHdG level and mtDNA deletion of gingival tissue and clinical parameters of periodontitis in the CP group

\begin{tabular}{lllllll}
\hline & $\begin{array}{l}\text { mtDNA } \\
\text { deletion }\end{array}$ & GI & PI & PD & CAL Age & $0.412^{*}$ \\
\hline 8 -OHdG & $0.932^{* *}$ & 0.105 & 0.185 & $0.517^{* *}$ & $0.527^{* *}$ & $0.372^{*}$ \\
\hline $\begin{array}{l}\text { mtDNA } \\
\text { deletion }\end{array}$ & - & -0.085 & 0.005 & $0.493^{* *}$ & $0.512^{* *}$ & \\
\hline
\end{tabular}

${ }^{*}$ Correlation is significant $(\mathrm{p}<0.05)$.

${ }^{* *}$ Correlation is significant $(\mathrm{p}<0.01)$.

Abbreviations: PI - plaque index, GI - gingival index, PD - probing depth, CAL - clinical attachment level. 
In addition, we found that both salivary $8-\mathrm{OHdG}$ level and the occurrence of the 5-kbp mtDNA deletion had significant positive correlations with age, PD, and CAL and no significant correlations with PI and GI in the periodontitis group. Generally, age-dependent oxidative mtDNA damage has been detected previously in liver (Yen et al. 1991), testis (Lee et al. 1994), muscle (Lee et al. 1994; Lim et al. 2000), heart (Katsumata et al. 1994), and lung (Lee et al. 1999) tissues of healthy elderly subjects. Lim et al. (Lim et al. 2000) clearly demonstrated that the frequency of oxidative mtDNA damage in the human skeletal muscle of end-stage renal disease (ESRD) patients was higher than in age-matched non-ESRD subjects and the increase in oxidative stress may lead to premature oxidative damage of the mitochondrial genome in diseased tissues. Suzuki et al. (Suzuki et al. 1999) reported that both the mtDNA deletion and the 8-OHdG levels correlated not only with age, but also with the duration of disease. In our previous study (Canakci et al. 2006) and in this study we did not find the mtDNA deletion in the gingival tissues of healthy subjects in the age group $<70$ years. This being so, the age factor may be related to the period of disease in periodontitis patients. Similarly, the positive correlation between oxidative DNA damage markers (mtDNA deletion and 8-OHdG level) and clinical parameters (PD and CAL) may be related to the duration of periodontitis and, indirectly, disease severity, because the long-term stimulation of cytokines such as TNF- $\alpha$ provokes both extracellular overproduction of ROS by PMN activation and a rise in ROS production from mitochondria. This hypothesis coheres with the characteristics of $\mathrm{CP}$.

It has been generally accepted that $8-\mathrm{OHdG}$ level increases in mitochondria due to single-strand DNA (mtDNA), which makes it more susceptible to damage. In a recent study, Nomoto et al. (Nomoto et al. 2008) concluded that cytoplasmic expression of $8-\mathrm{OHdG}$ reflects mtDNA damage in liver disease. The authors suggested that nuclear DNA damage needs stronger oxidative cellular damage than mitochondrial damage and their results indicated that mtDNA suffers oxidative stress earlier than nuclear DNA. Of course, the deletion of mtDNA may cause harmful effects on the tissues. The mtDNA deletion induces a vicious cycle of ROS generation. Because mtDNA encodes polypeptides involved in electron transport or ATP production, mtDNA damage will lead to a decrease in mitochondrial mRNA and mitochondrial protein synthesis. Loss of these mitochondrial proteins will lead to more generation of ROS, more mtDNA damage, and inhibition of ATP production. It is possible that the mtDNA deletion may cause tissue aging and increased cancer risk. Jessie et al. (Jessie et al. 2001) suggest that mtDNA deletions are not only a marker for aging, but also a cause of aging and cancer risk in the tissues.

In this study we first examined the oxidative DNA damage in periodontitis patients by measuring the salivary $8-\mathrm{OHdG}$ levels and investigating the mtDNA dele- tions of gingival tissues. In conclusion, our study demonstrated for the first time that the 5-kbp deleted mtDNA of gingival tissue was accumulated in parallel with an increased salivary $8-\mathrm{OHdG}$ level in periodontitis patients. Moreover, periodontitis patients with nondeleted mtDNA of the gingival tissue did not have significantly higher salivary $8-\mathrm{OHdG}$ levels than healthy controls. These results indicate that the increased salivary $8-\mathrm{OHdG}$ level might signify premature oxidative mtDNA damage. However, further investigations are needed to clarify the exact mechanism of oxidative DNA damage in diseased periodontium.

\section{REFERENCES}

Armitage GC (1999) Development of a classification system for periodontal diseases and conditions. Ann Periodontol $4: 1-6$

Canakci CF, Cicek Y, Canakci V (2005) Reactive oxygen species and human inflammatory periodontal diseases. Biochemistry 70:619-628

Canakci CF, Tatar A, Canakci V et al (2006) New evidence of premature oxidative DNA damage: mitochondrial DNA deletion in gingival tissue of patients with periodontitis. J Periodontol 77:1894-1900

Chapple IL, Matthews JB (2007) The role of reactive oxygen and antioxidant species in periodontal tissue destruction. Periodontol 2000 43:160-232

Chiou CC, Chang PY, Chan EC et al (2003) Urinary 8-hydroxydeoxyguanosine and its analogs as DNA marker of oxidative stress: development of an ELISA and measurement in both bladder and prostate cancers. Clin Chim Acta 334:87-94

Dincer Y, Erzin Y, Himmetoglu S et al (2007): Oxidative DNA damage and antioxidant activity in patients with inflammatory bowel disease. Dig Dis Sci 52:1636-1641

Jessie BC, Sun CQ, Irons HR et al (2001) Accumulation of mitochondrial DNA deletions in the malignant prostate of patients of different ages. Exp Gerontol 37:169-174

Kakimoto M, Inoguchi T, Sonta T et al (2002) Accumulation of 8-hydroxy-2'-deoxyguanosine and mitochondrial DNA deletion in kidney of diabetic rats. Diabetes 2002 51:1588-1595

Katsumata K, Hayakawa M, Tanaka M et al (1994) Fragmentation of human heart mitochondrial DNA associated with premature aging. Biochem Biophys Res Commun 202:102-110

Lee HC, Pang CY, Hsu HS et al (1994) Differential accumulations of 4977 bp deletion in mitochondrial DNA of various tissues in human ageing. Biochim Biophys Acta 1226:37-43

Lee HC, Lim ML, Lu CY et al (1999) Concurrent increase of oxidative DNA damage and lipid peroxidation together with mitochondrial DNA mutation in human lung tissues during aging-smoking enhances oxidative stress on the aged tissues. Arch Biochem Biophys 362:309-316

Lim PS, Cheng YM, Wei YH (2000) Large-scale mitochondrial DNA deletions in skeletal muscle of patients with end-stage renal disease. Free Radic Biol Med 29:454-463 
Liu H, Uno M, Kitazato KT et al (2004) Peripheral oxidative biomarkers constitute a valuable indicator of the severity of oxidative brain damage in acute cerebral infarction. Brain Res 1025:43-50

Liu TY, Chen CL, Chi CW (1996) Oxidative damage to DNA induced by areca nut extract. Mutat Res 367:25-31

Liu VW, Zhang C, Nagley P (1998) Mutations in mitochondrial DNA accumulate differentially in three different human tissues during ageing. Nucleic Acids Res 26:1268-1275

Löe H, Silness J (1963) Periodontal disease in pregnancy. I. Prevalence and severity. Acta Odontol Scand 21:531-551

Miller DR, Lamster IB, Chasens AI (1984) Role of the polymorphonuclear leukocyte in periodontal health and disease. $\mathbf{J}$ Clin Periodontol 11:1-15

Moseley R, Waddington RJ, Embery G (1997) Degradation of glycosaminoglycans by reactive oxygen species derived from stimulated polymorphonuclear leukocytes. Biochim Biophys Acta 1362:221-231

Nomoto K, Tsuneyama K, Takahashi H et al (2008) Cytoplasmic fine granular expression of 8-hydroxydeoxyguanosine reflects early mitochondrial oxidative DNA damage in nonalcoholic fatty liver disease. Appl Immunohistochem Mol Morphol 16:71-75

Ozmeric N (2005) Advances in periodontal disease markers. Clin Chim Acta 343:1-16

Sawamoto Y, Sugano N, Tanaka H et al (2005) Detection of periodontopathic bacteria and an oxidative stress marker in saliva from periodontitis patients. Oral Microbiol Immunol 20:216-220

Sculley DV, Langley-Evans SC (2002) Salivary antioxidants and periodontal disease status. Proc Nutr Soc 61:137-143

Sies H (1991) Oxidative stress; oxidants and antioxidants. Academic Press, New York

Silness J, Löe H (1964) Periodontal disease in pregnancy II. Correlation of between oral hygiene and periodontal condition. Acta Odontol Scand 22:121-135
Suzuki S, Hinokio Y, Kamatu K et al (1999) Oxidative damage to mitochondrial DNA and its relationship to diabetic complications. Diabetes Res Clin Pract 45:161-168

Takane M, Sugano N, Ezawa T et al (2005) A marker of oxidative stress in saliva: association with periodontally-involved teeth of a hopeless prognosis. J Oral Sci 47:53-57

Takane M, Sugano N, Iwasaki H et al (2002) New biomarker evidence of oxidative DNA damage in whole saliva from clinically healthy and periodontally diseased individuals. J Periodontol 73:551-554

Wada T, Tanji N, Ozawa A et al (2006) Mitochondrial DNA mutations and 8-hydroxy-2'-deoxyguanosine Content in Japanese patients with urinary bladder and renal cancers. Anticancer Res 26:3403-3408

Wallace DC, Shoffner JM, Trounce I et al (1995) Mitochondrial DNA mutations in human degenerative diseases and aging. Biochim Biophys Acta 1271:141-151

Wang AL, Lukas TJ, Yuan M et al (2008) Increased mitochondrial DNA damage and down-regulation of DNA repair enzymes in aged rodent retinal pigment epithelium and choroid. Mol Vis 14:644-651

Wu LL, Chiou CC, Chang PY et al (2004) Urinary 8-OHdG: a marker of oxidative stress to DNA and a risk factor for cancer, atherosclerosis and diabetics. Clin Chim Acta 339:1-9

Yakes FM, van Houten B (1997) Mitochondrial DNA damage is more extensive and persists longer than nuclear DNA damage in human cells following oxidative stress. Proc Natl Acad Sci USA 94:514-519

Yen TC, Su JH, King KL et al (1991) Ageing-associated 5 kbp deletion in human liver mitochondrial DNA. Biochem Biophys Res Commun 178:124-131 